\title{
Preparation of semi-IPN(BA-VAc-VAE) by emulsion polymerization and its properties investigation
}

\author{
SHI LUO, ZONGHUI LIU*, BAILING LIU* and QI LIU \\ Department of Polymer Chemistry and Physics, Chengdu Institute of Organic Chemistry, \\ The Graduate School of CAS, Chinese Academy of Sciences, Chengdu 610041, P.R. China \\ MS received 4 September 2008; revised 12 September 2010
}

\begin{abstract}
A semi-interpenetrating polymer network (semi-IPN) combining vinyl acetate-ethylene (VAE) and butyl acrylate (BA), as well as vinyl acetate (VAc), was prepared through emulsion polymerization with the help of self-made dispersant and diethylene glycol diacrylate (DEGDA), the cross-linking agent. Both the dispersant and DEGDA had significant contribution on the preparation of semi-IPN(BA-VAc-VAE). And, the DEGDA had a strong influence on the conversion of polymerization system. The effects of emulsifiers and their amounts on the stability of obtained emulsions have been determined, and the best choices have been made after series experiments. The optical microscopy (OM), FTIR and DSC methods were adopted to characterize the structure and composition, morphology, and $\mathrm{Tg}$ of the prepared emulsions, respectively. Various comparisons have been made between the semi-IPN and VAE emulsions, and the experimental results indicated that the semi-IPN(BA-VAc-VAE) had been developed with improved properties in both water resistance and adhesion.
\end{abstract}

Keywords. Vinyl acetate-ethylene; semi-interpenetrating polymer networks; emulsion polymerization; modification.

\section{Introduction}

Vinyl acetate-ethylene (VAE) emulsion is produced by copolymerization of vinyl acetate and ethylene under high pressure; usually, the vinyl acetate content ranges from 60 to $95 \%$, and ethylene from 5 to $40 \%$ of the total formulation. It should not be confused VAE with EVA copolymers, for the vinyl acetate content of EVA is below $40 \%$ (Chen et al 2001). EVA has a wide range of melt index values and good adhesion to a variety of materials, and its price is low (Benghalem et al 2003; Carmen et al 2003; Peon et al 2003; Rao et al 2003; Park et al 2006). However, it is not environmentally friendly because EVA has to be dissolved in organic solvents, such as benzene, trichlorobenzene, etc., before the use (Peon et al 2001; An et al 2003; Martinez-Garcia et al 2005).

VAE also offers considerable performance advantages due to its lower glass transition temperature (Tg) obtained through the incorporation of the ethylene in the main chain. As ethylene content increases, Tg of VAE decreases, rendering molecular chain of VAE more flexible. VAE also exhibits a lower surface tension and high temperature resistance. However, VAE has some disadvantages such as lack of depositing stability, poor water resistance and lower adhesion strength, etc. To improve

\footnotetext{
*Author for correspondence (zonghuiliu@ hotmail.com)
}

its related properties, many methods have been applied for VAE's modification; however, the method of interpenetrating polymer networks (IPNs) has been less reported.

IPNs are materials in which both components (and sometimes one only in semi-IPNs) are cross-linked during simultaneous or sequential polymerization processes. They have been investigated extensively, as evidenced by a number of reviews and monographs (Sperling et al 1970, 1994; Sperling 1985; Klempner and Berkowski 1987; Sperling and Mishra 1997). The phrase 'interpenetrating polymer network' was first coined by Millar in a study concerning ion-exchange materials containing sulfonated polystyrene (Millar 1960, 1962). IPNs possess several interesting characteristics in comparison with normal polyblends. Formation of IPNs is the only way of intimately combining cross-linked polymers, that will result in the mixture in a limited phase separation. Normal blending or mixing of polymers, however, will exhibit a multiphase morphology due to the thermodynamic incompatibility of blending components. But, if the mixing is accomplished simultaneously with crosslinking, phase separation may be kinetically controlled by permanent interlocking of entangled chains (Cassidy and Frisch 1985). IPN applications extend in fields such as semi-permeable membranes, ion-exchange resins, damping, toughening or impact-modifiers (Gauthier et al 2007). 
The aim of our research was to improve VAE's water resistance, adhesion and depositing stability. Here we choose BA and VAc as modifying monomers due to their hydrophobic nature and adhesion, respectively. For obtaining a better IPN, a cross-linking structure should be introduced within IPN. So, diethylene glycol diacrylate (DEGDA) was used as cross-linking agent owing to its long chain and high reactivity in lower reacting temperature.

\section{Experimental}

\subsection{Materials}

VAE-705 (Sinopec Sichuan Vinylon Works, 55\% of solid content), butyl acrylate (BA), vinyl acetate (VAc), acrylic acid (AA), ammonium persulfate (APS), polyoxyethylene alkylphenols (OP-10), sodium dodecyl sulfate (SDS), MS-1, fatty alcohol polyoxyethylene ether (OS-15) and DEGDA (diethylene glycol diacrylate, Tianjiao Chemical Co. Ltd) were used as received without further purification.

The dispersant, having a structure as shown below was prepared in our laboratory:

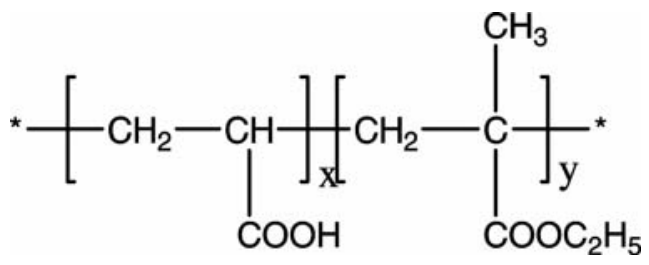

\subsection{Emulsion polymerization}

2.2a Preparation of semi-IPNs: The semi-IPNs were synthesized via emulsion polymerization method using water as reaction medium. All the syntheses were carried out under nitrogen atmosphere in a $250 \mathrm{ml}$ four-neck round-bottom flask equipped with reflux condenser, mechanical, stirrer and dropping funnels. The flask was heated using a water bath.

The basic recipes for the synthesis of semi-IPN were described in tables 1, 3 and 5 .

2.2b Preparation of $P(B A$ and $V A c)$ emulsions: The same method as described in $\$ 2.2 \mathrm{a}$ was used except without VAE.

\subsection{Characterization of semi-IPNs}

2.3a Solid content and polymerization conversion: The solid content and the polymerization conversion were measured by gravimetric analysis. A certain amount of latex was cast onto a petri dish and dried to a constant weight in an oven at $75-85^{\circ} \mathrm{C}$. The solid content and the final conversion were calculated by the following equations, respectively

$$
\text { Solid content }(\mathrm{wt} \%)=\frac{W_{2}-W_{0}}{W_{1}-W_{0}} \times 100 \%,
$$

where $W_{0}$ is the weight of the petri dish and $W_{1}$ and $W_{2}$ are the weight of latex before and after drying to the constant weight, respectively.

$$
\text { Conversion }(w t \%)=\frac{\text { Solid content }(w t \%) \times W_{3}}{W_{4}} \times 100 \%,
$$

where $W_{3}$ is the total weight of all the components added to the flask before polymerization and $W_{4}$ is the weight of materials except water.

2.3b Infrared spectra: Infrared spectra of the latex films were recorded with NICOLET MX-1E FT-IR spectrometer in the range 4000 to $400 \mathrm{~cm}^{-1}$.

2.3c Differential scanning calorimetry: The glass transition temperatures ( $\mathrm{Tg}$ ) of polymers were measured with a NETZSCH DSC 204 F1 differential scanning calorimeter at the acceleration rate of $10^{\circ} \mathrm{C} / \mathrm{min}$.

2.3d Water absorption ratio: The weighed latex films were dipped in distilled water for $24 \mathrm{~h}$. Then, the water on film surface was removed quickly with water-absorbing paper and the films were weighed again. The water absorption ratio of the films was calculated as follows:

Water absorption ratio $=\frac{W_{6}-W_{5}}{W_{6}} \times 100 \%$,

where $W_{5}$ and $W_{6}$ are the weight of the films before and after the water absorbing, respectively.

2.3e Adhesive strength: The initial adhesive of emulsions were examined by BLD-200S Electronic Peeling Machine, and $180^{\circ}$ peel strength test was referred to GB2792-81.

Table 1. Basic recipe for preparation of semi-IPN with various types of emulsifiers.

\begin{tabular}{lc}
\hline Ingredient & Amount $(\mathrm{g})$ \\
\hline $\mathrm{BA}$ & 21 \\
$\mathrm{VAc}$ & 15 \\
$\mathrm{VAE}-705$ & 11 \\
$\mathrm{AA}$ & 1.5 \\
DEGDA & $0 \cdot 42$ \\
APS & $0 \cdot 24$ \\
Emulsifier & $0 \cdot 84$ \\
$\mathrm{H}_{2} \mathrm{O}$ & 100 \\
\hline
\end{tabular}

Emulsifier (\%), $2 \%$ of total monomers weight, $80^{\circ} \mathrm{C}, 4 \mathrm{~h}$. 
Table 2. Stability of semi-IPN with different types of emulsifiers.

\begin{tabular}{lccccc}
\hline Emulsifier & OS-15 & MS-1 & OP-10 & SDS\&OP-10 & SDS\&OS-15 \\
\hline Stability & Demixing & Demixing & Stable & Demixing & Demixing \\
\hline
\end{tabular}

Table 3. Basic recipe for preparation of semi-IPN with varied amount of OP-10.

\begin{tabular}{lc}
\hline Ingredient & Amount $(\mathrm{g})$ \\
\hline BA & 21 \\
VAc & 15 \\
VAE-705 & 11 \\
AA & $1 \cdot 5$ \\
DEGDA & $0 \cdot 42$ \\
APS & $0 \cdot 24$ \\
OP-10 & See table 4 \\
$\mathrm{H}_{2} \mathrm{O}$ & 100 \\
\hline
\end{tabular}

DEGDA (\%), $1 \%$ of total monomers weight, $80^{\circ} \mathrm{C}, 4 \mathrm{~h}$.

2.3f Depositing stability: The depositing stability of emulsions were examined by maintaining them at room temperature, and then observed whether the emulsions were demixed.

2.3g Optical microscopy: The morphology of the emulsion films was observed with a JNOEC-MICROPHOTXSP24N optical microscopy (OM).

\section{Results and discussion}

\subsection{Effect of emulsifiers types on depositing stability}

Five series of experiments were designed according to the basic recipe listed in table 1 , where the emulsifiers were varied in each polymerization process, but their amount was kept constant. When obtained semi-IPNs were maintained at room temperature for $48 \mathrm{~h}$, the stabilities of them were listed in table 2, presenting the effects of diversified emulsifiers on the stabilities of semi-IPNs. From table 2, it can be found that OP-10 was a better emulsifier for the semi-IPNs, correspondingly. It was probably in respect to that, the dispersion and emulsion polymerization of the vinyl acetate particles might be attributed to the protective colloid formed by the nonionic emulsifier, such as OP-10.

\subsection{Effect of emulsifier amount on depositing stability}

Five series of experiments were designed according to the basic recipe listed in table 3 , where the amount of OP-10 varied in each polymerization process. When obtained semi-IPNs were maintained at room temperature for 1 month, the stabilities of them were observed and listed in table 4. The semi-IPN was stable when the content of
OP-10 was at least $6 \%$ of monomers. But more emulsifiers would make emulsions less adhesive, and so the mixed emulsifier system (OP-10 + dispersant) was adopted finally. The experimental result was possible related to the better dispersive effect of dispersant than OP-10.

\subsection{Effect of DEGDA amount on polymerization conversion}

Five series of experiments were designed according to the basic recipe listed in table 5, where the amount of DEGDA varied in respective polymerization. IPNs are materials where both components are cross-linked during the simultaneous or sequential polymerization processes. So, amount of the cross-linking agent, DEGDA, will have a vital effect on the semi-IPNs. The conversions of the emulsion polymerization with various amount of DEGDA are shown in figure 1. It is shown that with increasing the amount of DEGDA in the emulsion polymerization, the conversion increased. The maximum conversion was achieved when $2 \%$ DEGDA was used, and then the conversion of system decreased with the increase of DEGDA introduced. This result was possibly attributed to the increase of system's viscosity at a far higher concentration in the system.

\subsection{Structure and composition of semi-IPN emulsion}

In the IR spectrum of the emulsion film shown in figure 2. The characteristic absorption of the $\mathrm{C}-\mathrm{H}$ bond of vinyl acetate is at $2933 \mathrm{~cm}^{-1}$. The peak of $1236 \mathrm{~cm}^{-1}$ is the characteristic absorption of the $-\mathrm{COO}-$ group. The peak of $1371 \mathrm{~cm}^{-1}$ and $1021 \mathrm{~cm}^{-1}$ are also the characteristic absorption of vinyl acetate. In the IR spectrum of emulsion film shown in figures 2 (a) and (b), the strong absorption of acrylate is at $1163 \mathrm{~cm}^{-1}$, and the peak of $942 \mathrm{~cm}^{-1}$ is the characteristic absorption of butyl acrylate. From the IR spectrum, we can see that the semi-IPN has components of VAE and P(BA-VAc).

\subsection{The glass transition temperatures and morphology of emulsion films}

From figure 3 , we can see that the VAE emulsion had one $\mathrm{Tg}$ around $2 \cdot 9^{\circ} \mathrm{C}$. The semi-IPN emulsion also had only one $\mathrm{Tg}$ of $-18 \cdot 7^{\circ} \mathrm{C}$. The experimental results indicated that the semi-IPN was the copolymer with a complete compatibility between all the components. 
Table 4. Effect of varied amount of OP-10 on emulsion stability.

\begin{tabular}{lccccc}
\hline Amount & $2 \%$ & $3 \%$ & $4.5 \%$ & $6 \%$ & $2+1.5 \%$ Dispersant (self-made) \\
\hline Stability & Demixing & Demixing & Demixing & Stable & Stable \\
\hline
\end{tabular}

Table 5. Basic recipe for preparation of semi-IPN with varied amount of DEGDA.

\begin{tabular}{lc}
\hline Ingredient & Amount $(\mathrm{g})$ \\
\hline $\mathrm{BA}$ & 21 \\
$\mathrm{VAc}$ & 15 \\
$\mathrm{VAE}-705$ & 11 \\
$\mathrm{AA}$ & $1 \cdot 5$ \\
DEGDA & See figure 1 \\
APS & $0 \cdot 24$ \\
$\mathrm{OP}-10$ & $0 \cdot 84$ \\
$\mathrm{H}_{2} \mathrm{O}$ & 100 \\
\hline
\end{tabular}

OP- $10 \%, 2 \%$ of total monomers weight, $80^{\circ} \mathrm{C}, 4 \mathrm{~h}$.

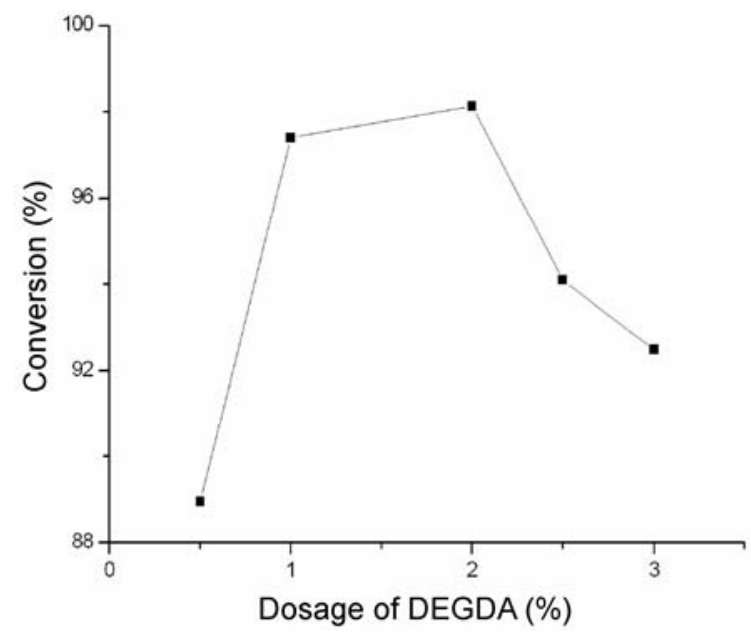

Figure 1. System conversion of varied DEGDA ratio.

The OM micrograph in figure 4(a) shows that $\mathrm{P}(\mathrm{BA}-$ VAc) had entered into the interior of VAE with a good compatibility. It was evidence of the formation of IPNs.

\subsection{Water resistance of emulsion films}

Five series of experiments were designed according to the recipe listed in table 6 . And the effect of BA/VAc ratio on water resistance of emulsion films was listed in table 7. The water absorption ratio of VAE film was very high, implying its poor water resistance. However, the water absorption ratio of the semi-IPN was very low. It was because the network structure of the semi-IPN become tighter with the effect of cross-linking agent. In addition, the water absorption ratio of the semi-IPN decreased with the increase of BA/VAc ratio. It was because of BA which was more hydrophobic than VAc.

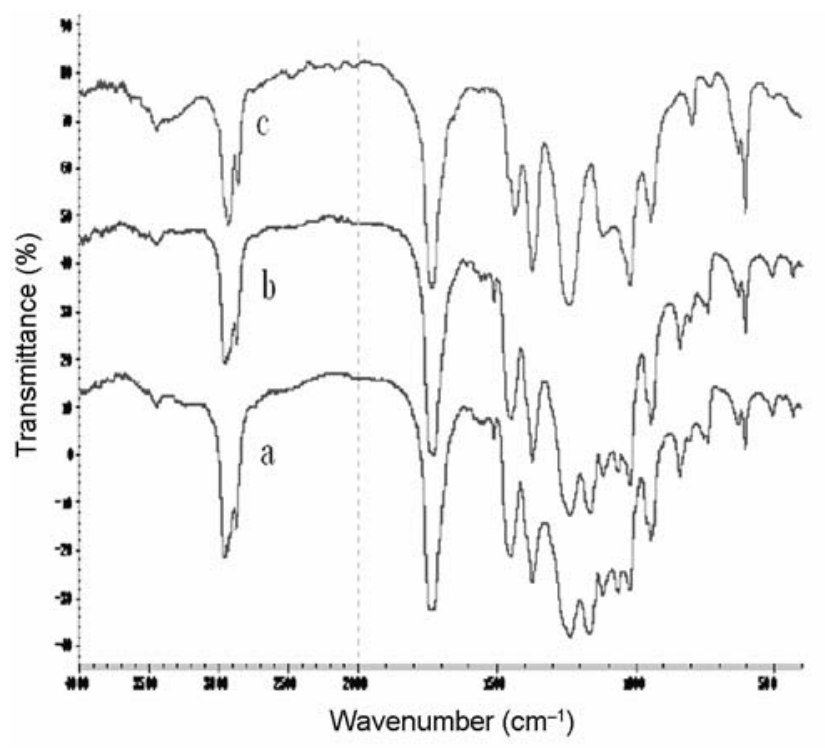

Figure 2. IR spectrum of the emulsion films: (a) $\mathrm{P}(\mathrm{BA}-\mathrm{VAc})$; (b) semi-IPN and (c) VAE.

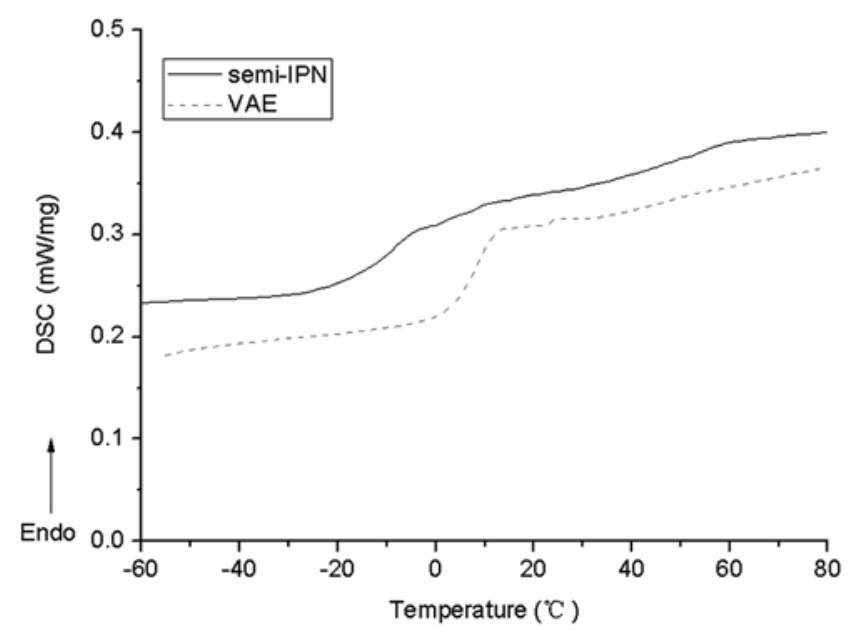

Figure 3. DSC analysis of emulsion films: semi-IPN and VAE.

\subsection{Adhesion of emulsions}

Five series of experiments were designed according to the recipe listed in table 6 . And the effect of BA/VAc ratio on adhesion of emulsions was shown in table 7. We can see that because of the addition of BA and VAc, the adhesion, especially the initial adhesion of the semi-IPN, has been improved remarkably in comparison with the original VAE 

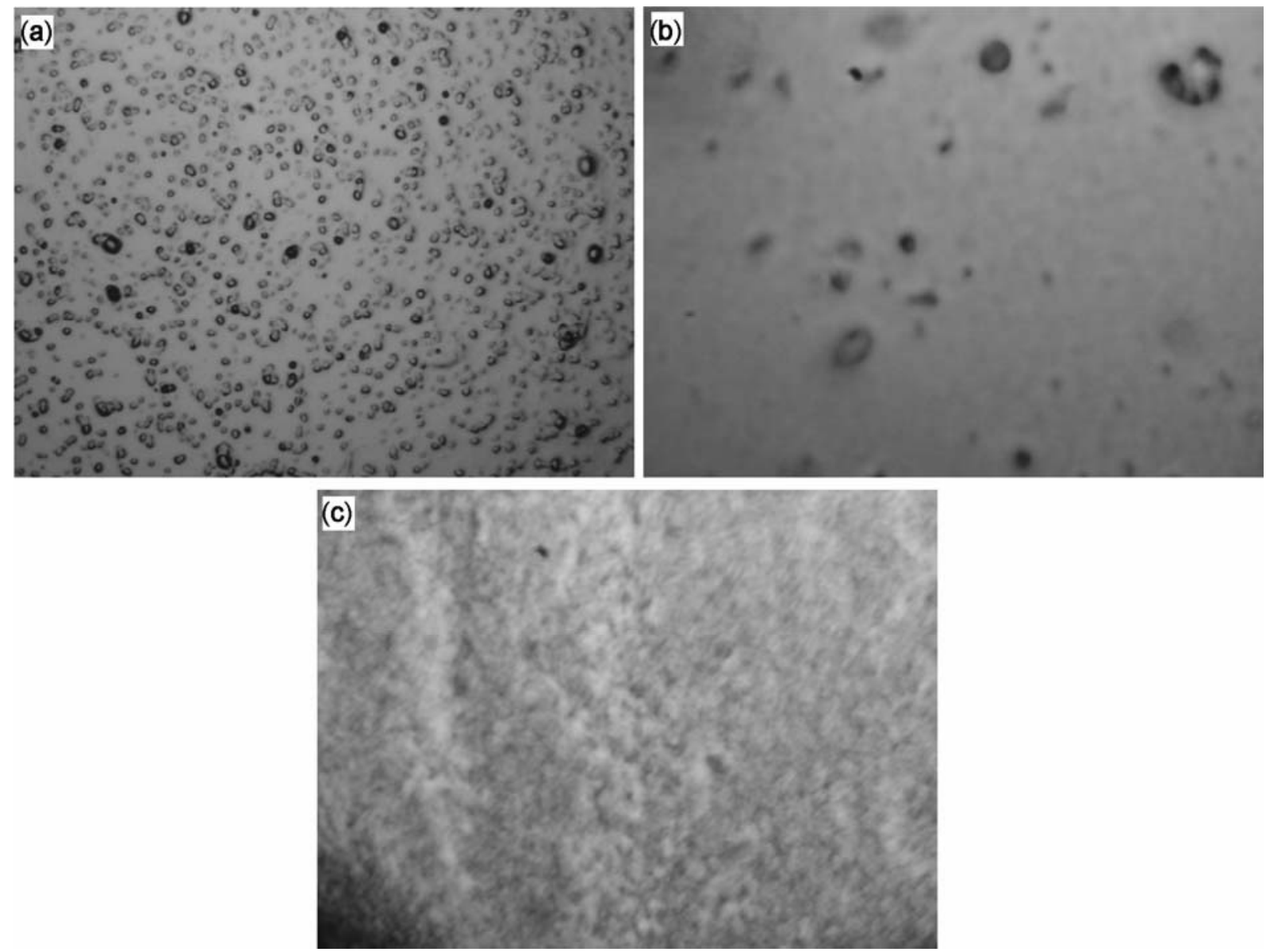

Figure 4. OM micrograph of emulsion films: (a) semi-IPN; (b) P(BA-VAc) and (c) VAE.

Table 6. Basic recipe for preparation of semi-IPN with varied BA/VAc ratio.

\begin{tabular}{lcccc}
\hline Ingredient & Amount $(\mathrm{g})$ & Amount $(\mathrm{g})$ & Amount $(\mathrm{g})$ & Amount $(\mathrm{g})$ \\
\hline $\mathrm{BA}$ & 24 & 21 & 18 & 15 \\
VAc & 12 & 15 & 18 & 21 \\
VAE-705 & 11 & 11 & 11 & 11 \\
$\mathrm{AA}$ & $1 \cdot 5$ & $1 \cdot 5$ & $1 \cdot 5$ & $1 \cdot 5$ \\
DEGDA & $0 \cdot 42$ & $0 \cdot 42$ & $0 \cdot 42$ & $0 \cdot 42$ \\
APS & $0 \cdot 24$ & $0 \cdot 24$ & $0 \cdot 24$ & $0 \cdot 24$ \\
OP-10 & $0 \cdot 84$ & $0 \cdot 84$ & $0 \cdot 84$ & $0 \cdot 84$ \\
Dispersant & $0 \cdot 63$ & $0 \cdot 63$ & $0 \cdot 63$ & $0 \cdot 63$ \\
$\mathrm{H}_{2} \mathrm{O}$ & 100 & 100 & 100 & 100 \\
\hline
\end{tabular}

OP- $10 \%, 2 \%$ of total monomers weight, DEGDA $\%, 2 \%$ of total monomers weight, $80^{\circ} \mathrm{C}, 4 \mathrm{~h}$.

Table 7. Water absorption of emulsion films.

\begin{tabular}{lccccc}
\hline Types & $\mathrm{BA}: \mathrm{VAc}=2: 1$ & $\mathrm{BA}: \mathrm{VAc}=7: 5$ & $\mathrm{BA}: \mathrm{VAc}=1: 1$ & $\mathrm{BA}: \mathrm{VAc}=5: 7$ & $\mathrm{VAE}$ \\
\hline Water absorption ratio $(\%)$ & 10.63 & 12.58 & 13.87 & $15 \cdot 14$ & 28.76 \\
\hline
\end{tabular}

Table 8. Adhesion of emulsions.

\begin{tabular}{lccccc}
\hline Types & $\mathrm{BA}: \mathrm{VAc}=2: 1$ & $\mathrm{BA}: \mathrm{VAc}=7: 5$ & $\mathrm{BA}: \mathrm{VAc}=1: 1$ & $\mathrm{BA}: \mathrm{VAc}=5: 7$ & $\mathrm{VAE}$ \\
\hline Initial adhesion $(\mathrm{N} / 25 \mathrm{~mm})$ & $3 \cdot 8$ & $3 \cdot 5$ & $3 \cdot 3$ & $2 \cdot 9$ & $0 \cdot 5$ \\
$180^{\circ}$ peel strength $(\mathrm{N} / 25 \mathrm{~mm})$ & $3 \cdot 1$ & $4 \cdot 0$ & $5 \cdot 2$ & $6 \cdot 0$ & $2 \cdot 5$ \\
\hline
\end{tabular}


emulsion. With the decrease of $\mathrm{BA} / \mathrm{VAc}$ ratio, the initial adhesion and $180^{\circ}$ peel strength of the semi-IPN reduced and increased, respectively. It was because $\mathrm{Tg}$ of BA was lower than VAc, resulting in the better initial adhesion of BA. With the decrease of BA/VAc ratio, more VAc can enhance the rigidity and intensity of the film of semi-IPN emulsion. Accordingly, the $180^{\circ}$ peel strength of the semi-IPN increased with reducing BA/VAc ratio.

\section{Conclusion}

Semi-interpenetrating polymer networks (semi-IPNs) combining VAE, BA and VAc had been obtained by emulsion polymerization through the proper amount of DEGDA and self-made emulsifier systems (OP-10 + dispersant) were introduced in system. The amount of used emulsifiers can be decreased evidently because of the use of dispersant. The conversion of emulsion polymerization was influenced by the amount of DEGDA. The morphology of semi-IPN could be observed by OM micrographs. The DSC and FT-IR analytic results had presented the degree of phase consistence. In comparison with VAE, the water resistance and adhesion of semi-IPN had been improved greatly. And, the semi-IPN emulsion could keep stable within 1 year at least.

\section{References}

An Q F, Qian J W, Sun H B, Wang L N, Zhang L and Chen H L 2003 J. Membr. Sci. 222113
Benghalem A, Oughilas A and Leboukh A 2003 Polymer 44 4381

Carmen M, Cepeda-Jimenez, Torregrosa-Macia R and MartinMartinez J M 2003 Surf Coat. Technol. 174-175 94

Cassidy E and Frisch H L 1985 Polym. Eng. Sci. 25758

Chen X M, Zhong H, Jia L Q, Tang R G, Qiao J L and Zhang Z Y 2001 Int. J. Adhesion \& Adhesives 21221

Gauthier C, Plesse C, Vidal F, Pelletier J- M, Chevrot C and Teyssie D 2007 Polymer 487476

Klempner D and Berkowski L 1987 Interpenetrating polymer networks. In Encyclopedia of polymer science and engineering (ed.) J L Kroschwitz (New York: Wiley) Vol. 8, pp 279341

Martinez-Garcia A, Sanchez-Reche A and Martin-Martinez J M 2005 Int. J. Adhesion \& Adhesives 25201

Millar J R 1960 J. Chem. Soc. 1311

Millar J R 1962 J. Chem. Soc. 1789

Park Y J, Joo, H -S, Kim H -J and Lee Y -K 2006 Int. J. Adhesion \& Adhesives 26571

Peon J, Vega J F, Aroca M and Matinez-Salazar J 2001 Polymer 428093

Peon J, Vega J F, Del A B and Martinez-Salazar J 2003 Polymer 442911

Rao P S, Subrahmanya S and Sathyanarayana D N 2003 Synth. Metals 139397

Sperling L H 1985 Polym. Eng. Sci. 25517

Sperling L H and Mishra V 1997 In IPNs around the world: science and engineering (eds) S C Kim and L H Sperling (New York: Wiley) pp 1-25

Sperling L H, George H F and Huelck V 1970 J. Appl. Polym. Sci. 142815

Sperling L H, Klempner D and Utracki L A (eds) 1994 Interpenetrating polymer networks (Washington: American Chemical Society) 